\title{
Electroacupuncture attenuates depressive disorder and gastrointestinal dysfunction in myocardial hypertrophy via upregulation of 5-HT on Rats
}

Jia Li

Hubei University of Chinese Medicine https://orcid.org/0000-0003-0444-2263

Wei Lu

Hubei University of Chinese Medicine

Wei Huang

Hubei University of Chinese Medicine

Song Wu

Hubei University of Chinese Medicine

\section{Yimeng Fu}

Hubei University of Chinese Medicine

Jianyi Chen

Hubei University of Chinese Medicine

Zhongsheng Huang

Hubei University of Chinese Medicine

Xiaohong Zhou

Hubei College of Chinese Medicine

Hua Wang ( $\nabla 8209009 @ q q . c o m$ )

Hubei University of Chinese Medicine

\section{Research}

Keywords: Myocardial hypertrophy, Electroacupucture, 5-hydroxytryptamine, Gastrointestinal motility

Posted Date: August 18th, 2020

DOI: https://doi.org/10.21203/rs.3.rs-57478/v1

License: (c) (i) This work is licensed under a Creative Commons Attribution 4.0 International License. Read Full License

Version of Record: A version of this preprint was published at Acupuncture \& Electro-Therapeutics Research on January 1st, 2021. See the published version at 
https://doi.org/10.3727/036012921X16287835103444. 


\section{Abstract}

Background: Acupuncture therapy has been described as a preventive and therapeutic technique with low or no risk for a number of conditions related to cardiovascular disease. However, its role in protecting against myocardial hypertrophy $(\mathrm{MH})$ has not been elucidated. This study aimed to investigate whether electroacupuncture (EA) can attenuate $\mathrm{MH}$ by upregulating tryptophan 5-hydroxytryptamine $(5-\mathrm{HT})$ metabolism, depressive disorder and gastrointestinal dysfunction in $\mathrm{MH}$ rats model.

Methods: We assessed the anti-myocardial ischemia effects of EA by using the isoproterenol hydrochloride (ISO)-induced myocardial hypertrophy (MH) rats model. Forty 3-month-old Sprague-Dawley (SD) rats were randomly divided into normal, model, EA and sham EA groups, with ten rats in each group. Regression of $\mathrm{MH}$ was evaluated by ratio of heart weight/body weight (HW/BW) and ratio of left ventricular weight/body weight (LVW/BW). Ultrastructural observation and hematoxylin and eosin (H\&E) staining were evaluated to cardiac pathology. The sucrose preference test (SPT), open field test (OFT), and forced swim test (FSW) were conducted to examine the depression-like behavior. Gastric retention and intestinal transit were evaluated using the carbon powder labeling method, while immunohistochemical staining was used to detect 5-HT expression in the cerebral cortex and ileum.

Key findings: EA therapy alleviates rats' depression-like symptoms. For example, the rate of sucrose solution consumption was higher, the total and central distance traveled in the open field test were increased, and immobility time was decreased in these rats by EA therapy. Furthermore, swimming, struggling and latency to immobility were increased in the forced swim test as well. Expression of 5-HT was increased in the cerebral cortex and ileum after EA treatment.

Conclusion: EA improved the ischemic and depressed symptoms, as well as gastrointestinal dysfunction of $\mathrm{MH}$ rats model induced by ISO, which may related with 5-HT metabolism.

\section{Highlights}

1. The current study investigates the effects of electroacupucture $(E A)$ attenuates $\mathrm{MH}$ by upregulating tryptophan 5-hydroxytryptamine (5-HT) metabolism, depressive disorder and gastrointestinal dysfunction in $\mathrm{MH}$ rats model.

2. Abnormal 5-HT expression in the intestinal tract plays a crucial role in the presentation of depression after $\mathrm{MH}$, and is provides a theoretical basis for acupuncture treatment of $\mathrm{MH}$.

\section{Background}

Myocardial hypertrophy $(\mathrm{MH})$, a heart muscle thickening that causes the enlargement of ventricles, is the primary reaction of myocardium in response to mechanical overload or stress [1]. Depression is a frequent psychiatric symptom among patients with $\mathrm{MH}$ [2] and it effects the prognosis of $\mathrm{MH}$ as an independent risk factor. In addition to this, previous studies showed that $\mathrm{MH}$ has an influence on intestinal barrier integrity and somatic psychiatric symptoms (e.g., gastrointestinal symptoms, fatigue, 
poor appetite, and nausea) that also reflects prognosis of the disease. It was demonstrated in the clinical studies that myocardial injury was reduced effectively by acupuncture pretreatment in patients with myocardial ischemia [3]. Meanwhile, according to a lot of experimental studies, results indicated that acupuncture can meaningfully diminish the incidence rates of sudden death [4], arrhythmias, and angina pectoris [5]. However, there is no research to explore that acupuncture can regulate the association between the mechanisms of somatic and psychiatric symptoms after $\mathrm{MH}$ at present, especially to focus on 5-Hydroxytryptamine (5-HT), which is a pivotal neurotransmitter in the central nervous system. Meanwhile, most of 5-HT in the body is contained within gastrointestinal tract, and it is important for depression and gastrointestinal function.

In the early animal research [6], we observed that EA can improve cardiac function in $\mathrm{MH}$ rats by modulating upstream neuroendocrine cytokines that regulate the extracellular signal-regulated kinase signaling pathways. For further research, we hypothesize that electroacupuncture (EA) regulates depression and gastrointestinal disorders after $\mathrm{MH}$ by ameliorating central and enteric 5 -HT dysfunction.

\section{Materials And Methods}

\section{1 Chemicals and reagents}

Rabbits anti-mouse 5-HT antibody and goat anti-rabbit secondary antibody were purchased from Invitrogen and Abcam (MA1-81821 and ab150077; California USA and Cambridge UK respectively).

\subsection{Animals groups and Models}

All experimental procedures comply with the guidelines of National Institutes of Health (NIH) "Guide for the Care and Use of Laboratory Animals and the legislation of the People's Republic of China for the use and care of laboratory animals. The experimental protocols were approved by the Animal Experimentation Ethics Committee of the Hubei University of Traditional Chinese Medicine, China. A total of forty 3-month-old female Sprague-Dawley (SD) rats, weighing $200 \mathrm{~g}$ to $220 \mathrm{~g}$, were provided by Tongji Medical College of HUST (SCXK (E) No. 2016-0057). In accordance with the random number table, rats were divided into four groups which are normal, model, EA and sham EA groups, with ten rats in each group. Rats in the normal group received $3 \mathrm{mg} / \mathrm{kg} \cdot \mathrm{d}$ of physiologic saline injection into the back skin, whereas the other rats were injected with isoproterenol hydrochloride (ISO), as previously described [6].

\subsection{EA stimulation}

The acupuncture stimulation involved the insertion of stainless steel needles $(0.2 \mathrm{~mm}$ in diameter and $13.0 \mathrm{~mm}$ in length) into the acupoint PC6 at a vertical depth of 2-3 $\mathrm{mm}$ in groups EA and sham EA. Needles were manually twisted for one minute at a frequency of about $2 \mathrm{~Hz}$ before EA treatment. Rats in EA group received EA stimulation on bilateral acupoint PC6 with the following stimulus parameters: continuous-wave, frequency of $2 \mathrm{~Hz}$, and current intensity of $1 \mathrm{~mA}$, while rats in sham EA group received no electric current. They were treated for 20 min every day for 14 days. 


\subsection{Evaluation of regression of cardiac ischemia and cardiac hypertrophy}

Reversion of cardiac hypertrophy was assessed by ratio of heart weight/body weight (HW/BW) and ratio of left ventricular weight/body weight (LVW/BW). Regression of cardiac ischemia was recorded by electrocardiogram (ECG) recording electrode resettlement as performed previously [6]. For recording the standard $\mathbb{B}$-lead ECG, we used BL-420 physiological function experiment system. Its negative and positive stainless steel electrodes were placed beneath the skin close to bilateral left and right forelimb horizontal grain respectively, and the reference electrode was placed beneath the skin of the right hind limb. In process of recording, we selected a resolution ratio of $500 \mathrm{nv} / \mathrm{mv}$, chart speed of $50 \mathrm{~ms} / \mathrm{div}$, and taken ten cardiac cycle into calculation.

\subsection{Ultrastructure observation}

Rats were quickly decapitated after anesthesia with intraperitoneal injection of $10 \%$ chloral hydrate (100 $\mathrm{g} / 0.3 \mathrm{ml})$, and cardiac tissues were separated. The central ventricle muscle of left ventricular free wall was removed and chopped into $1 \mathrm{~mm} \times 1 \mathrm{~mm} \times 1 \mathrm{~mm}$ size, fixed in $2.5 \%$ glutaraldehyde for 48 hours, then in $1 \%$ osmium tetrachloride for 1.5 hour, dehydration, embedding, sectioning, and examined on Hitachi $\mathrm{H}$ 600 transmission electron microscopy (Hitachi Company, Tokyo, Japan).

\subsection{Infarct size measurement}

Rats were euthanized and hearts were removed after 2 weeks modeling. In order to histological assessment, a small fragment from each heart was fixed in $10 \%$ buffered formalin solution and dehydrated with alcohol and xylene. After dehydration, each sample was embedded in paraffin wax and divided into slides of $5 \mu \mathrm{m}$ thickness with a Leica rotation microtome. Groups of slides were stained with hematoxylin and eosin (H\&E) based on standard methods. Areas of myocardial infarction were observed using ImageJ software (NIH, Bethesda, MD, USA).

\subsection{Sucrose preference test}

Rats were single placed in a quiet room with two bottles of sucrose solution $(1 \%, w / v)$ for the first $24 \mathrm{~h}$ period. Then one bottle was replaced with tap water for the second $24 \mathrm{~h}$ period. After this initial adaption phase, rats were deprived of food and water for $24 \mathrm{~h}$ and then permitted access to two bottles for $3 \mathrm{~h}$, one containing $100 \mathrm{ml}$ of $1 \%$ sucrose solution and the other $100 \mathrm{ml}$ of tap water. Sucrose preference was defined as the ratio of consumption of sucrose solution and the consumption of both pure water and sucrose solution during the test phase, and calculated as follows: percentage $(\%)=$ total sucrose consumption / (total sucrose consumption + total water consumption) $\times 100 \%$.

\subsection{Forced swim test}

Briefly, each rat was subjected to pre-swimming habituation for 15 minutes on day 15 and tested 24 hours later. Rats were individually placed into plexiglas cylinders (internal diameter $21 \mathrm{~cm}$; height $50 \mathrm{~cm}$ ) filled with water (depth $30 \mathrm{~cm} ; 25^{\circ} \mathrm{C}$ ) for 5 min of forced swimming in the training session. Time spent on 
swimming, striving, and immobile and the latency to immobility were recorded, using a swimming analysis system (EthoVision XT, Noldus, the Netherlands) to analyze.

\subsection{Open-field test}

Briefly, rats were placed individually at center of the field and were allowed to explore any areas freely in open field test. Then, behavior was recorded using a detection system (JLBehv-LAR-1; Shanghai Jiliang Software Technology Co., Ltd., Shanghai, China). The total and central distances explored during the 5minute test were used to analyzed the behaviors.

\subsection{Determination of gastric residual and gastrointestinal transit rates}

Before commencing the experiment, animals were deprived of food for $24 \mathrm{~h}$ but allowed free access to water. Each rat was received $1 \mathrm{~mL} / 100 \mathrm{~g}$ nutritional black semi-solid paste and was sacrificed $20 \mathrm{~min}$ later. Afterward, opening the abdominal cavity, stomach and small intestine were excised after ligation of the pylorus and the cardia, then the stomach was obtained, dried, and weighed. It was cut along the greater curvature, the contents were rinsed, and the stomach was weighed again. The difference in gastric weight showed the weight of the gastric residues. The gastric residual rate was calculated as follows: gastric residual rate $(\%)=$ (total gastric weight - gastric net weight) $/$ intragastric volume $\times 100$.

Immediately after excision of the stomach, the small intestine (from the pylorus to the ileocecum) was completely freed from its mesenteric attachments. Its total length and the carbon propulsion distance were measured by a meter scale. The gastrointestinal transit rate was calculated as follows: intestine propulsion rate $(\%)=$ carbon propulsion distance $/$ total length of small intestine $\times 100 \%$.

\subsection{Immunofluorescence staining of 5-HT}

The rat brain and terminal ileum was removed and sections ( $5 \mu \mathrm{m}$ thick) were obtained consecutively. The sections were washed with phosphate-buffered saline (PBS), and processed with green-fluorescent Alexa Fluor $488(1: 500)$ to incubate. The nuclear marker DAPI was used. The fluorescence microscope (Leica, Germany) was used to observed and imaged these sections. SABC method was used to perform immunohistochemical staining of 5-HT protein. Positive staining of protein was shown in the cytoplasm. NeuN-positive cells were counted in three random images from each section by a technician blinded to this study.

\subsection{Statistical analysis}

Data were presented as mean \pm standard error of mean (S.E.M) in this study. Unpaired student's t-test was used to analyze two sets of data. Differences among groups were tested using one way or repeated measures analysis of variance (ANOVA). The Tukey-Kramer test was used to differentiate means. A $P$ value of less than 0.05 was considered statistically significant.

\section{Results}




\subsection{EA prevented cardiac hypertrophy induced by pressure overload}

We first determined the degree of intervention for cardiac hypertrophy induced by ISO after treatment with EA for two weeks. Results in fig. 1 show that the ratios of HW/BW and LVW/BW were evidently increased in groups model and sham EA compared to the group Control, which were meaningfully suppressed by EA treatment $(P<0.05$, Fig. $1 A$ and $1 B)$. ECG analysis detected that $S T$ segment and $R-R$ interval was decreased in cardiac hypertrophic rats treated with EA compared with those rats in model and sham EA groups $(P<0.05$, Fig. $1 C, 1 D$ and $1 E)$.

Ultrastructural of cardiac cell was observed by transmission electron microscopy. In model group, the cardiac cell exhibited mitochondria swelling, excessive cell apoptosis, endothelial cell interstitial hyperplasia, muscle plasma nets expansion, out[1]sync contraction of contraction band, and intercalated disc deformation. Compared EA group with model and sham EA groups, myocardial ischemia and hypertrophy was improved. Ultrastructure observation suggested that treatment with EA significantly reduced pressure overload-induced increase cardiomyocyte cross-sectional areas ( Fig. 1F).

Histological observation of control group (after H\&E staining) presented that cardiac muscle cells were arranged in order, the nucleus was clearly visible and myocardial fibers were compact. However, rats in model group showed a great number of structurally fiber tissues were disordered, with widened gaps and local fibrosis. Cells were ruptured and exhibited dissolution and a distribution disorder in more than $50 \%$ of the area of ischemic necrosis, which indicated that myocardial cells were minimally damaged. Sham EA group did not obviously differ from the model group on histological evaluation. In contrast, compared to model group, the cardiac muscle of EA group presented obvious improvement, with lighter myocardial fibrosis, smaller nuclei and significantly reduced areas of ischemia ( Fig. 1G).

\subsection{Sucrose preference test results}

A one-way analysis of variance exhibited significant differences in sucrose intake among 4 groups at 2 weeks modeling $(F[3,36]=23.84 ; P<0.01)$. In addition, Tukey's multiple comparisons suggested that sucrose intake in model and sham EA groups was decreased relative to normal group (both $P<0.01$ ). However, EA increased the sucrose intake while compared with groups model and sham EA (both $P<$ 0.01). There were no significant differences between model and sham EA groups ( $P \otimes 0.05$, Fig $2 B)$.

\subsection{Open-field test results}

A one-way analysis of variance showed significant differences in the total and central distance among 4 groups $(F[3,36]=63.34, P<0.01 ; F[3,36]=303.2, P<0.01$, respectively). Further Tukey's multiple comparisons showed that the total and central distances were significantly reduced in model and sham EA groups relative to normal group (both $P<0.01$ ). However, EA increased the total and central distances while compared with groups model and sham EA (both $P<0.01$ ). There were no significant differences between model and sham EA groups ( $P \otimes 0.05$, Fig $2 \mathrm{C}$ and $2 \mathrm{D})$.

\subsection{Forced swim test results}


A one-way analysis of variance revealed significant differences in immobility time, swimming time, struggling time, and latency to immobility among 4 groups $(F[3,36]=11.44, P<0.01 ; F[3,36]=58.41, P$ $<0.01 ; \mathrm{F}[3,36]=77.94, \mathrm{P}<0.01 ; \mathrm{F}[3,36]=137.8, \mathrm{P}<0.01$ respectively). Further Tukey's multiple comparisons showed that immobility time was prolonged, whereas swimming and struggling time and latency to immobility were reduced in model and sham EA groups relative to the normal group (all $\mathrm{P}<$ 0.01). Compared to normal and sham EA groups, EA group exhibited reduced immobility time and enhanced swimming and struggling time and latency to immobility (all $\mathrm{P}<0.05$ ). There were no significant differences in immobility time, swimming time, struggling time and latency to immobility between model and sham EA groups (all $\mathrm{p} \otimes 0.05$; Fig $2 \mathrm{E}-2 \mathrm{H}$ ).

\subsection{Analysis of gastric residual and gastrointestinal transit rates}

A one-way analysis of variance revealed significant differences in the gastric residual and intestinal transit rates among 4 groups $(F[3,36]=26.41, P<0.01 ; F[3,36]=37.73, P<0.01$, respectively $)$. Further pairwise comparisons showed that the gastric residual and intestinal transit rates were increased in model and sham EA groups relative to normal group (all $P<0.01$ ). Compared to normal and sham EA groups, EA group exhibited reduced gastric residual and intestinal transit rates (all $P<0.05)$. There were no significant differences between sham and normal groups (both $\mathrm{p} \otimes 0.05$; Fig $3 \mathrm{~A}$ and $3 \mathrm{~B}$ ).

3.8 Immunohistochemical detection of 5-HT expression in the cerebral cortex region and the terminal ileum

A one-way analysis of variance showed significant differences in 5-HT expression in the cerebral cortex region and the distal ileum among 4 groups $(F[3,36]=13.77, P<0.01 ; F[3,36]=15.38, P<0.01$, respectively). Further pairwise comparisons showed that $5-\mathrm{HT}$ expression in the cerebral cortex and the distal ileum was significantly decreased in model and sham EA groups relative to EA group ( all $P<0.05$ ). Compared to normal and sham EA groups, EA group exhibited increased 5-HT expression in the cerebral cortex region and the distal ileum (all $\mathrm{P}<0.05$ ). There were no significant differences between model and sham EA groups (both $\mathrm{p} \otimes 0.05$; Fig $4 \mathrm{C}$ and 4D).

\section{Discussion}

Ischemic heart disease is known to be one of the major causes of death in the world and it can directly lead to myocardial infarction [7]. Modern study has demonstrated that EA possesses therapeutic effects on cardiovascular system [8]. Previous research has indicated that acupoint PC6 was preferred and considered effective in treating cardiovascular disorders, which can attenuate cardiac injury, through regulating heart rate variability [9], apoptosis [10], and myocardial ischemia [11]. Our previous researches had confirmed that EA could effectively promote myocardial fibrosis and protect myocardial tissue against ischemia injury [6]. In the rats with ISO induced MH, we found a depression of ST segment of the ECG, which is indicative of ischaemia, repeated EA at PC6 for 20 min a day for 14 days improved cardiac function in MH rats model. Moreover, according to results of SPT, OFT and FWT, we observed that EA could significantly ameliorate depression-like symptoms. In addition, the gastric retention rate and 
gastrointestinal transit rate were improved, and expression of 5-HT was increased in the cerebral cortex and ileum after EA treatment. Depressive disorder and gastrointestinal dysfunction after $\mathrm{MH}$ are almost accompanied by abnormal 5-HT metabolism. This provides a new mind for why the somatic, cognitive, and psychiatric symptoms of depression commonly appear after $\mathrm{MH}$.

Cardiovascular disease, including ischemic heart disease and cerebrovascular disease, has been identified as a leading provider to both mortality and disease burden globally [12]. Furthermore, depression may bring about autonomic nervous system disorders such as decreased heart rate variability, increased heart rate, excessive heart rate response to physical stress, and increased ventricular depolarization variability, thereby increasing the risk of cardiovascular disease [13]. $\mathrm{MH}$ is a potential target for therapy to improve outcomes among depressed non-ST elevation acute coronary syndrome patients [14], with high rates of morbidity and mortality and is often comorbid with gastrointestinal dysfunction [15]. Remarkably 5-HT, it is considered as a good communicator between central nervous system (CNS) and peripheral system, because of its signal transporting role in CNS transduction when releasing by presynapic vesicles, and it plays an important role in regulating emotional station and depression [16]. More and more evidence reveals that the decline of prefrontal cortex 5-HT release is highly contribute to etiology of depression and anxiety as some suicide victims [17]. Depression, the most prevalent mood disorder, is characterized by low mood, lack of motivation, reduced energy, anhedonia and despair. In this study, we assessed the degree of depressive-like behavior after $\mathrm{MH}$ in rats. Sucrose preference test was an indicator of anhedonia-like behavioral change, the open field test was a way to evaluate exploratory activity, and the forced swimming reflected the degree of despair. Through our experiment, we discovered that, after $\mathrm{MH}$, the preference of sucrose was notably decreased and the total and central distance traveled in the open field test were remarkably decreased as well. Furthermore, immobility time in forced swimming test was increased, and swimming time and latency to immobility were shortened. Changes in 5-HT were assayed immunchemistry with fluorescence detection. 5-HT in cerebral cortex and terminal ileum tissues were significantly decreased in $\mathrm{MH}$ rats model, wile EA treatment on PC6 increased 5-HT expression both in cerebral cortex and terminal ileum. Meanwhile, these data from this study are correspond to the 5-HT hypothesis of depression. To the best of our knowledge, the current study is the first to report that EA can improve $\mathrm{MH}$ by regulating $5-\mathrm{HT}$ expression both in brain and gastrointestinal.

In our body, $95 \%$ of $5-\mathrm{HT}$ is synthesized in the intestinal tract and $90 \%$ is distributed to intestinal chromaffin cells, whereas the remaining $5 \%$ is found in the enteric nervous system [18]. The enteric cavity stimulates to secret $5-\mathrm{HT}$ in a stressful environment, which acts on intrinsic primary afferent neurons in the mucosal membrane. These afferent neurons can activate interneurons in the dorsal horn and initiate intestinal tract movement through receptor pathways [19]. Increases in 5-HT have associated with gastrointestinal motility dysfunction in pathological state. Low metabolism of 5-HT brings about abnormalities in brain regions linked with learning, memory, and emotion. It functions through the binding of its receptors, including the G-protein coupled 5-HT1A receptor, which is a predominant contributor to the regulation of emotion [20]. 


\section{Conclusion}

In summary, taking the viewpoint that acupuncture therapy is of some notable value for $\mathrm{MH}$, we believe that there is a close relationship between PC6 (acupoint) and heart (organ). In summary, 5-HT acts as a bridge between gut and brain and is an critical regulator involved in EA regulation. Abnormal 5-HT expression in the intestinal tract plays a crucial role in presentation of depression after $\mathrm{MH}$, and is provides a theoretical basis for acupuncture treatment of $\mathrm{MH}$.

\section{Abbreviations}

5-HT 5-Hydroxytryptamine

CNS Central Nervous System

EA Electroacupucture

ECG Electrocardiogram

H\&E Hematoxylin and Eosin

HW/BW Heart Weight/Body Weight

ISO Isoproterenol Hydrochloride

LVW/BW Left Ventricular Weight/Body Weight

$\mathrm{MH} \quad$ Myocardial Hypertrophy

S.E.M Standard Error of Mean

\section{Declarations}

\section{Ethics approval and consent to participate}

All experimental procedures comply with the guidelines of National Institutes of Health (NIH) "Guide for the Care and Use of Laboratory Animals and the legislation of the People's Republic of China for the use and care of laboratory animals. The experimental protocols were approved by the Animal Experimentation Ethics Committee of the Hubei University of Traditional Chinese Medicine, China.

\section{Consent for publication}

Not applicable.

\section{Conflict of Interests}


The authors declare no conflict of interests regarding publication of this paper.

\section{Availability of data and materials}

The datasets used or analysed during our current study are available from the corresponding author on reasonable request.

\section{Competing interests}

The authors declare no conflict of interests regarding the publication of this paper.

\section{Funding}

This study was funded by the project grant from NSFC (Natural Science Foundation of China, No: 81804165 and 81973931$)$.

\section{Authors' contributions}

Jia Li and Hua Wang conceived and designed the experiments. Song Wu, Wei Huang and Wei Lu performed the experiments. Yimeng Fu analyzed the data. Jia Li contributed reagents/materials/analysis tools and wrote the paper. Zhongsheng Huang and Jianyi Chen helped revise the manuscript. All authors approved the final version of the manuscript accepted for publication.

\section{Acknowledgements}

We appreciated Bocun Li for revising drawings in the manuscript. This study was funded by the project grant from NSFC (Natural Science Foundation of China, No: 81804165 and 81973931).

\section{Data Availability Statement}

The data used to support the findings of this study are included within the article.

\section{References}

1. Gladka MM, da Costa Martins PA, De Windt LJ. Small changes can make a big difference - microrna regulation of cardiac hypertrophy. J Mol Cell Cardiol. 2012; 52: 74-82.

2. Barth J, Schumacher $M$, Herrmann-Lingen $C$. Depression as a risk factor for mortality in patients with coronary heart disease: a meta-analysis. Psychosom Med. 2004;66(6):802-13.

3. Wang Q., Liang D., Wang F., et al. Efficacy of electroacupuncture pretreatment for myocardial injury in patients undergoing percutaneous coronary intervention: a randomized clinical trial with a 2-year follow-up. International Journal of Cardiology. 2015;194:28-35.

4. Mehta P. K., Polk D. M., Zhang X., et al. A randomized controlled trial of acupuncture in stable ischemic heart disease patients. International Journal of Cardiology. 2014;176(2):367-374. 
5. Longhurst J. C. Electroacupuncture treatment of arrhythmias in myocardial ischemia. American Journal of Physiology-Heart and Circulatory Physiology. 2007;292(5):H2032-H2034.

6. Li J, Li J, Liang F, et al. Electroacupuncture at PC6 (Neiguan) Improves Extracellular Signal-Regulated Kinase Signaling Pathways Through the Regulation of Neuroendocrine Cytokines in Myocardial Hypertrophic Rats. Evid Based Complement Alternat Med. 2012;2012:792820.

7. Takahashi M. NLRP3 inflammasome as a novel player in myocardial infarction. Int Heart J 2014; 55 : 101-5.

8. Middlekauff HR. Acupuncture in the treatment of heart failure. Cardiol Rev 2004, 12(3):171-3.

9. Wang G, Tian Y, Jia S, et al. Acupuncture regulates the heart rate variability. J Acupunct Meridian Stud. 2015 Apr;8(2):94-8.

10. Wang Y, Wang W, Li D, et al. The beneficial effect of electro-acupuncture given at PC6 (Neiguan-point) by the increase in cardiac transient outward $\mathrm{K}+$ current channel which depends on the gene and protein expressions in artificially induced myocardial ischemia rats. Acupunct Electrother Res. 2014;39(3-4):259-73.

11. Wang S, Ren L, Jia L, et al. Effect of acupuncture at Neiguan (PC 6) on cardiac function using echocardiography in myocardial ischemia rats induced by isoproterenol. J Tradit Chin Med. 2015 Dec;35(6):653-8.

12. Mackay J, Mensah GA, Mendis S, et al. The atlas of heart disease and stroke. Geneva: World Health Organization; 2004.

13. Carney RM, Freedland KE, Veith RC. Depression, the autonomic nervous system, and coronary heart disease. Psychosom Med 2005;67 Suppl 1:S29-33.

14. Whang W, Davidson KW, Palmeri NO, et al. Relations among depressive symptoms, electrocardiographic hypertrophy, and cardiac events in non-ST elevation acute coronary syndrome patients. Eur Heart J Acute Cardiovasc Care. 2016 Sep;5(5):455-60.

15. Marques FZ, Nelson E, Chu PY, et al. High-Fiber Diet and Acetate Supplementation Change the Gut Microbiota and Prevent the Development of Hypertension and Heart Failure in Hypertensive Mice. Circulation. 2017 Mar 7;135(10):964-977.

16. Zhang $L J$, Zeng XT, Zhao MJ, et al. The important effect of 5-HTTLPR polymorphism on the risk of depression in patients with coronary heart disease: a meta-analysis. BMC Cardiovasc Disord. 2020 Mar 18;20(1):141.

17. Yohn CN, Gergues MM, Samuels BA. The role of 5-HT receptors in depression. Mol Brain. 2017 Jun 24;10(1):28.

18. Gershon MD. Serotonin is a sword and a shield of the bowel: serotonin plays offense and defense. Trans Am Clin Climatol Assoc. 2012;123: 268-280.

19. Liu HN, Ohya S, Nishizawa Y, et al. Serotonin augments gut pacemaker activity via 5-HT3 receptors. PLoS One. 2011;6: e24928 10.1371/journal.pone.0024928. 
20. Arnold SA, Hagg T. Serotonin $1 A$ receptor agonist increases species- and region-selective adult CNS proliferation, but not through CNTF. Neuropharmacology. 2012; 63: 1238-1247.

\section{Figures}
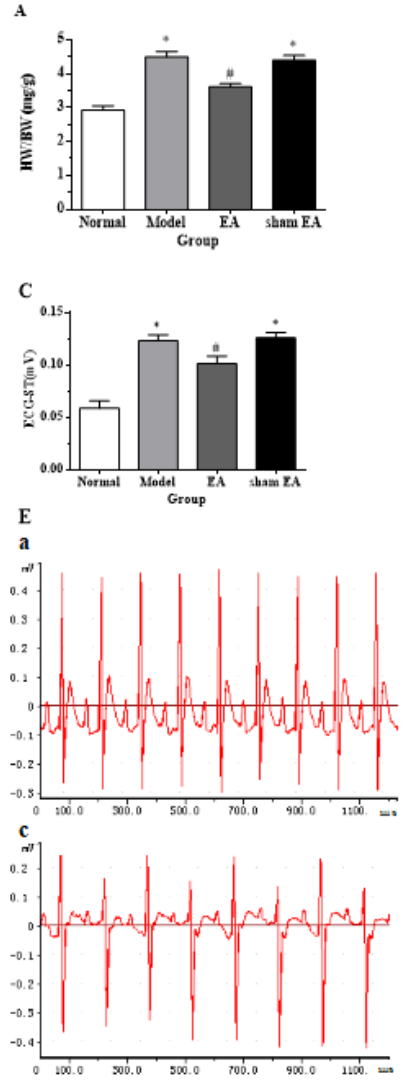
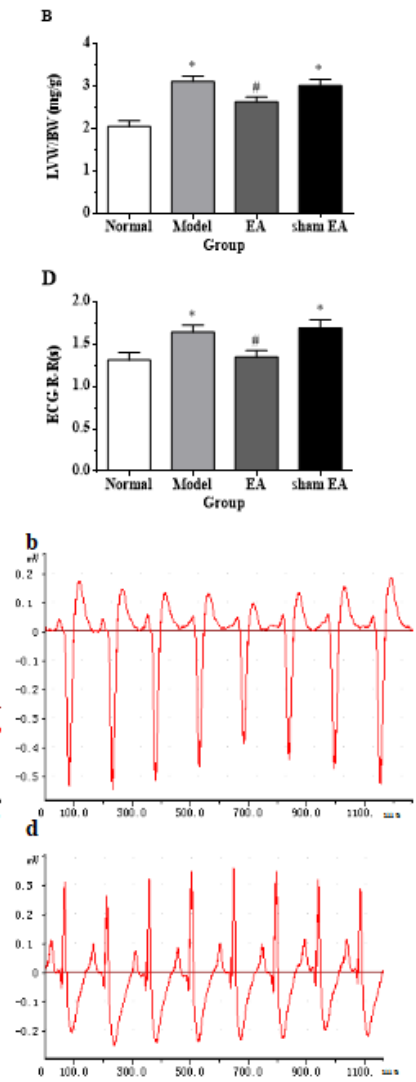

F
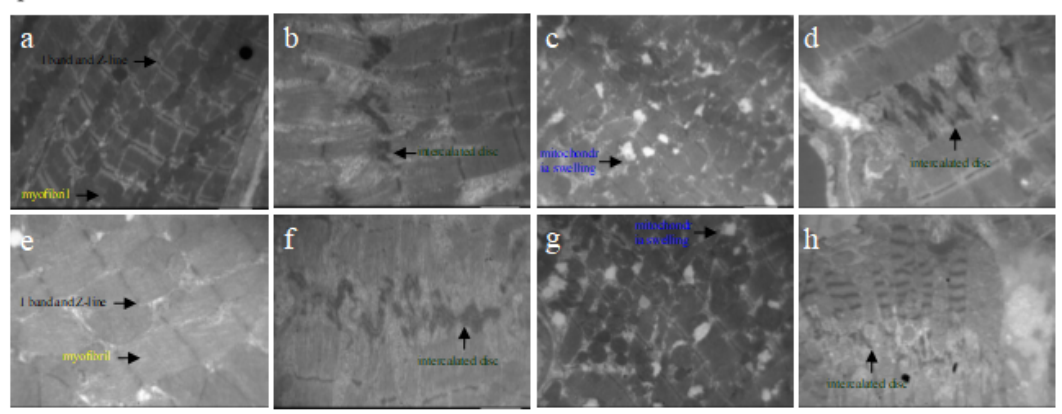

G

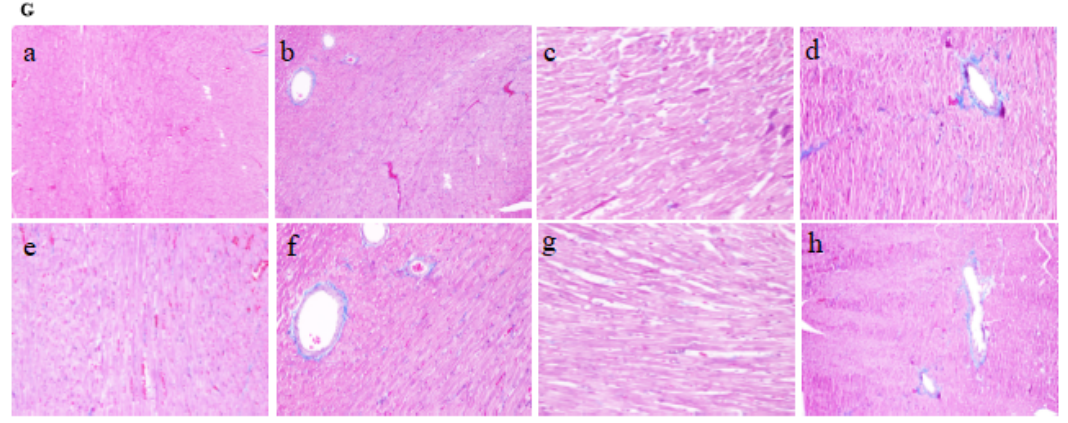

\section{Figure 1}

Effect of EA on cardiac hypertrophy induced by pressure overload. (A) Changes in the ratio of heart weight to body weight (HW/BW) and the ratio of left ventricular weight to body weight (LVW/BW). (B) Quantification of ST segment and RR interval during electrocardiogram (ECG). (C) Successful establishment of ISO induced MH defined by the observation of an ST-segment elevation of higher than $0.5 \mathrm{mV}$ for more than 30 minutes (lead II) and an increased R-wave amplitude as well as R-R interval on electrocardiography. In group electroacupuncture (EA), ST-segment elevation and R-wave amplitude decreased while compared with groups model and sham EA (Fig 1). Data were presented as means \pm S.E.M, ${ }^{*}<<0.05$ vs. normal group; $\# P<0.05$ vs. model group, $n=10$. (E) Representative $E C G$ of the control group (a), model group (b), EA group (c) and sham EA group (d). (F) Ultrastructure observation of left ventricular free wall, including mitochondria swelling, cell apoptosis and the arrangement of myogenic fibers. With reference to normal group, compared electroacupuncture (EA) group with model group, EA significantly reduced cardiac muscular tissue injury. Ultrastructure features in normal group ( $a \times 17000$,

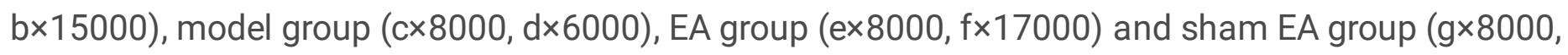
$h \times 12000) .(G)$ Effect of electroacupuncture $(E A)$ on area of myocardial infarction in rats with myocardial 
hypertrophy $(\mathrm{MH})$. Histological observation after hematoxylin and eosin (H\&E) staining in the control group $(a, b)$, model group $(c, d)$, EA group $(e, f)$ and sham EA group $(g, h)$. All images are $\times 200$ magnification.

A Control Model $\quad$ EA sham EA

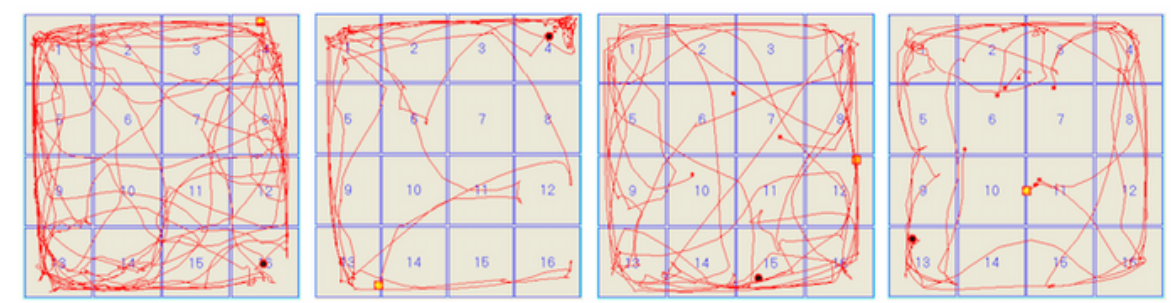

B

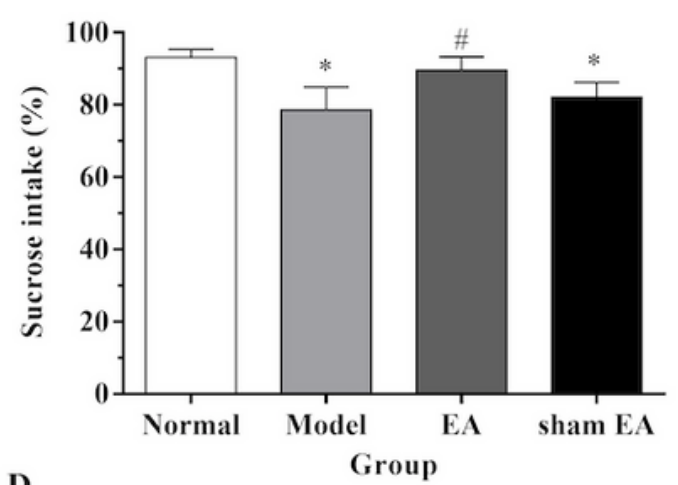

D

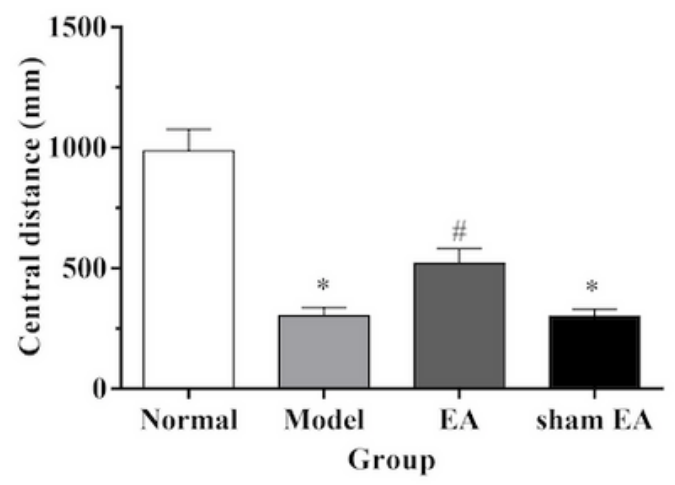

C

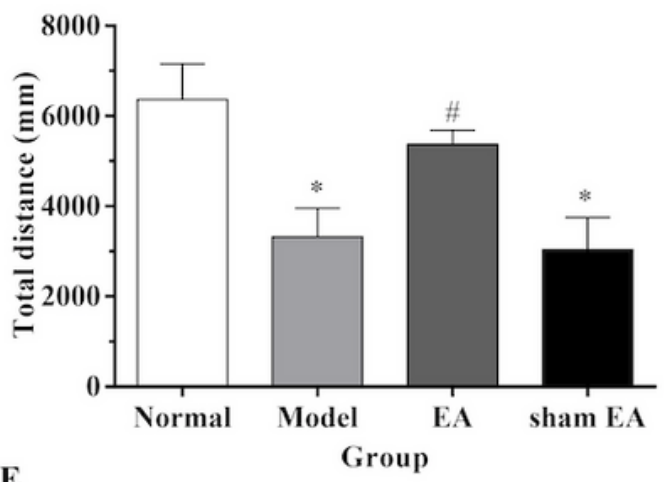

E

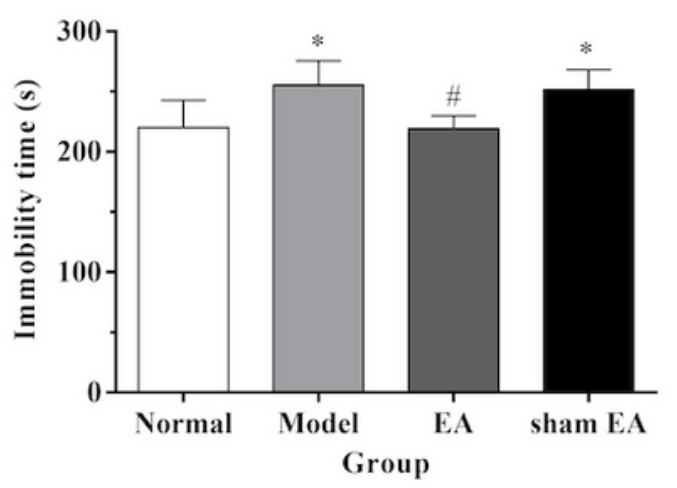

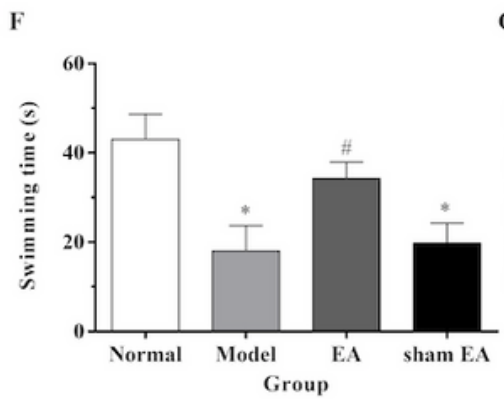

G

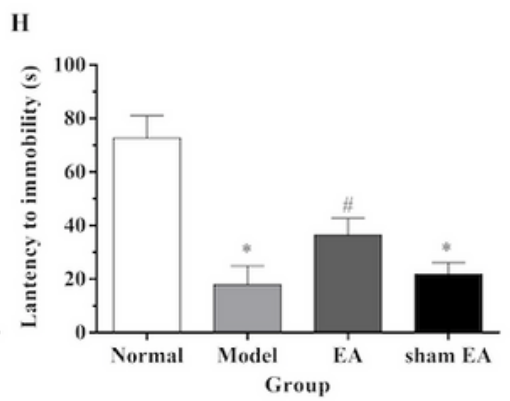

Figure 2

EA regulates behavioral measures. (A) Representative images of the observed rat behaviors in the open field test (OFT) and forced swim test (FST). (B) Sucrose intake in the 4 groups. (C-D) Total and central 
distance in the 4 groups. $(\mathrm{E}-\mathrm{H})$ Immobility, swimming time, struggling time, and latency to immobility in the 4 groups. Data are presented as means \pm S.E.M ( $n=10$ per group). ${ }^{*} P<0.05$ vs. normal group; \# $P<$ 0.05 vs. EA group.

A

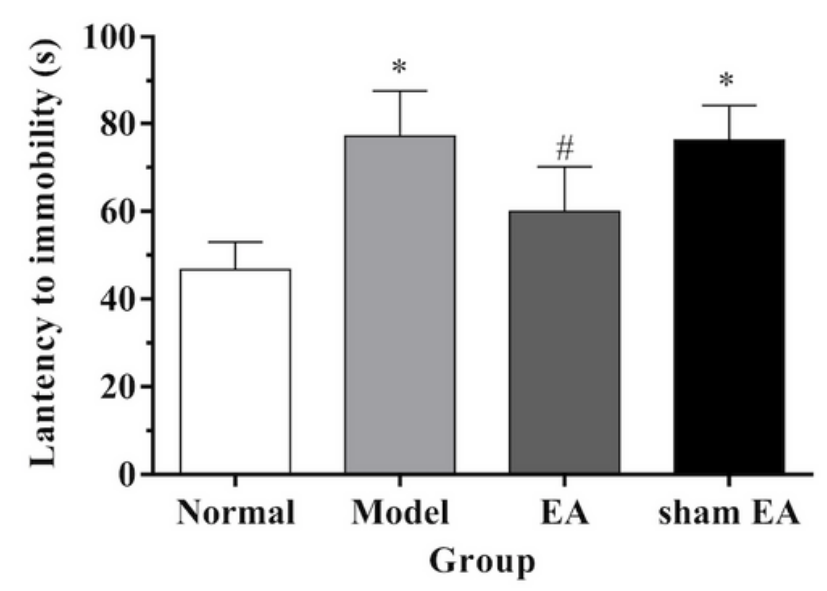

B

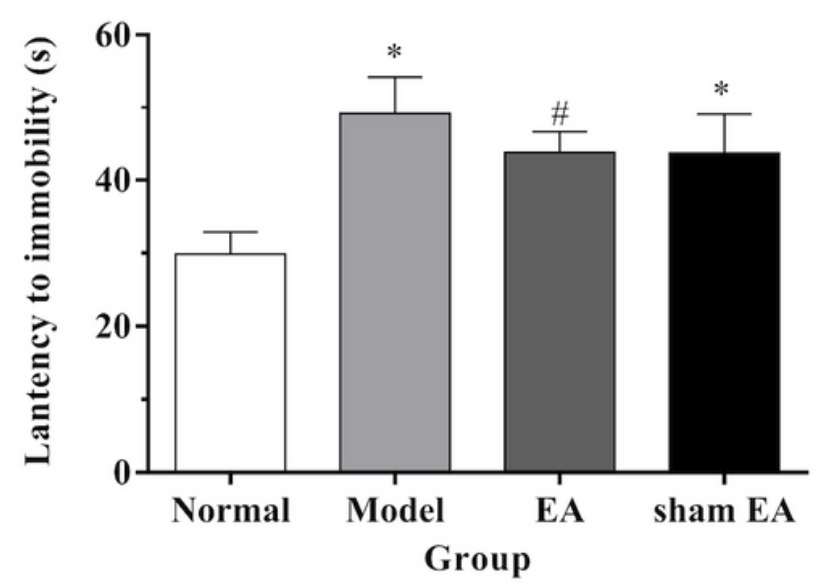

Figure 3

EA improves gastric retention and intestinal transit. Gastric retention $(A)$ and intestinal transit $(B)$ measured 2 weeks after the myocardial hypertrophy $(\mathrm{MH})$ and electroacupucture $(\mathrm{EA})$ treatment. Data are presented as means \pm S.E.M. $\left(n=10\right.$ per group). ${ }^{*} P<0.05$ vs. normal group; $\# P<0.05$ vs. EA group. 

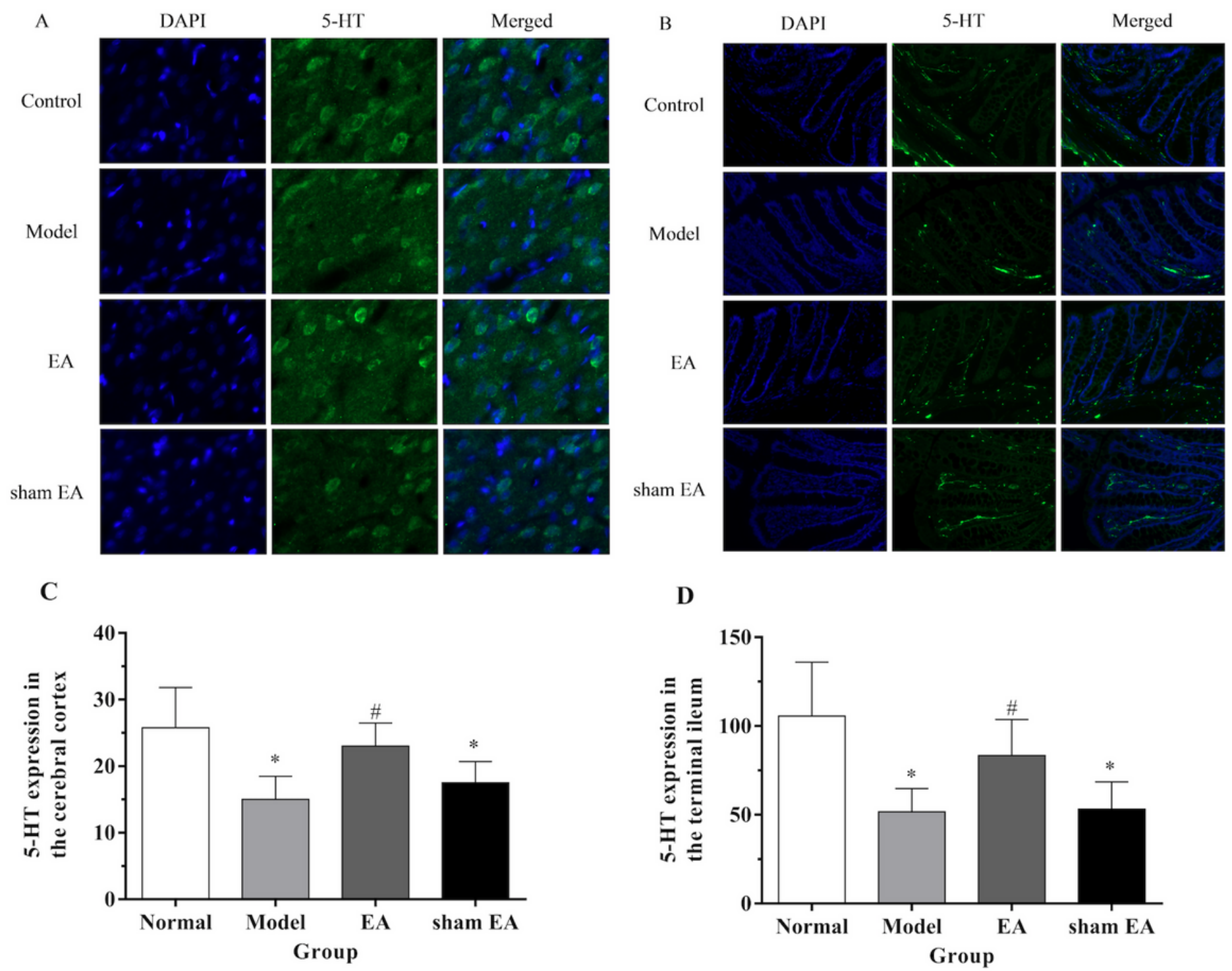

D

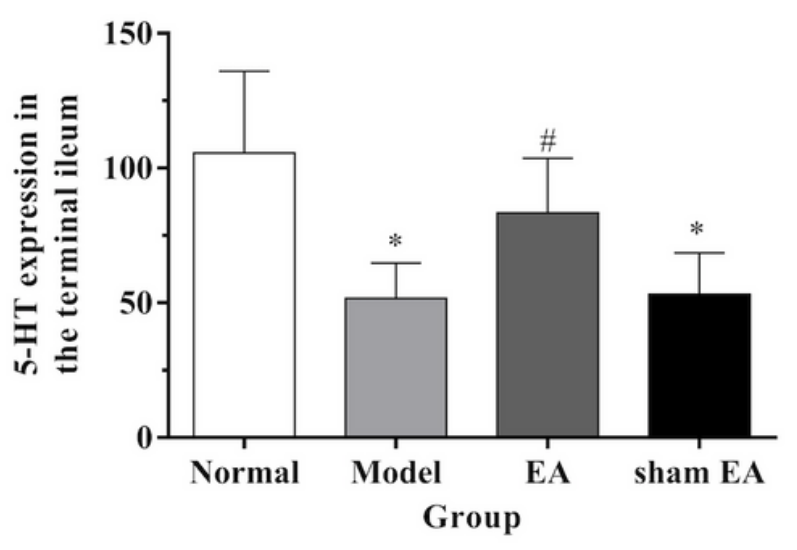

\section{Figure 4}

EA regulates $5-\mathrm{HT}$ activity in cerebral cortex and terminal ileum tissues $(\mathrm{x} 100$, immunofluorescence staining). A to D: 5-Hydroxytryptamine (5-HT) immunostaining of rat cerebral cortex tissues and terminal ileum in normal, model, electroacupucture (EA) and sham EA groups, respectively. Immunostaining showing decreased 5-HT expression in model group compared with normal group. Acupuncture treatment further enhanced 5-HT expression expression. *Significantly lower 5-HT expression in model group compared to normal group $(P<0.05)$. \#Significantly higher 5 -HT expression in rats in EA group compared with model and sham EA groups $(P<0.05)$. Values are presented as means \pm S.E.M $(n=10)$. ${ }^{*}<0.05$, vs. normal group; $\# \mathrm{P}<0.05$, vs. model and sham EA groups. 